\title{
The Relationship Between Bone Mineral Density and Type 2 Diabetes in Obese Children and Adolescents at the Time of Initial Diagnosis
}

Authors

Hae Sang Lee ${ }^{1}$, Jong Seo Yoon ${ }^{1}$, Kyu Jung Park' ${ }^{1}$ Jung Sub Lim², Jin Soon Hwang ${ }^{1}$

Affiliations

1 Department of Pediatrics, Ajou University School of Medicine, Ajou University Hospital, Suwon, Korea

2 Department of Pediatrics, Korea Cancer Center Hospital, Seoul, Korea

Key words

bone mineral density, type 2 diabetes, diabetic complications

received 11.04 .2018

accepted 02.10 .2018

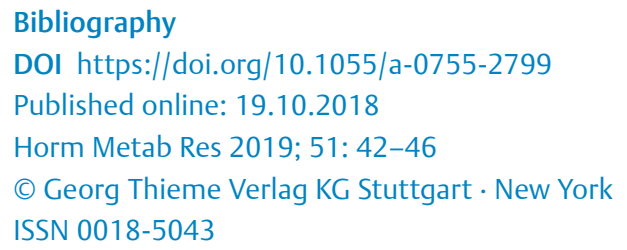

\section{ABSTRACT}

Long-term effects of type 2 diabetes mellitus (T2D) on bone health remain unclear. The objective of this study was to assess the possible association of bone mineral density (BMD) at multiple sites with T2D after correcting for several potential confounders such as age, sex, Tanner stage, and BMI known to affect BMD in adolescents with newly developed T2D. In this cross-sectional study, 17 children and adolescents with T2D and 59 age, sex, and BMI-matched controls were included. All subjects underwent dual-energy X-ray absorptiometry to measure regional and whole-body composition with Lunar Prodigy at the time of initial diagnosis. A BMD Z-score was calculated using data from healthy Korean children and adolescents after adjusting for height-for-age. The mean age of all subjects was $12.9 \pm 2.4$ years (range, $8.3-18.3$ years). $\mathrm{BMD}_{\mathrm{ht}}$ Z-scores for lumbar spine and total body after adjusted for age, sex, BMI SDS, and Tanner stage were not significantly different between patients and controls. However, $\mathrm{BMD}_{\mathrm{ht}} \mathrm{Z}$-scores for femur neck and bone mineral apparent density (BMAD) Z-scores of lumbar spine were significantly lower in T2D patients than those in healthy controls. HOMA-IR or HbA1c was not associated with $\mathrm{BMD}_{\mathrm{ht}} \mathrm{Z}$-scores at multiple sites. $\mathrm{BMD}_{\mathrm{ht}}$ Z-scores at multiple sites except femur neck in adolescents with newly developed T2D were similar to those in obese controls after adjustment for potential confounders.

\section{Introduction}

The increasing prevalence of childhood obesity is a worldwide problem [1]. In 2011, among Korean children and adolescents, 11.6\% of boys and $10.9 \%$ of girls were obese as defined by a body mass index (BMI) of $25 \mathrm{~kg} / \mathrm{m}^{2}$ or greater [2]. During the past 5 years, the number of overweight children in Korea has more than doubled and the prevalence of type 2 diabetes mellitus (T2D) has increased dramatically in children and adolescents.

Complications such as retinopathy, nephropathy, and neuropathy are well known to exist in patients with T2D. Recent studies on T2D have been done on other organ systems such as the skeletal system [3]. Although decreased bone mineral density (BMD) is consistently observed in patients with type $1 \mathrm{DM}$, the relationship between T2D and bone density remains unclear in adults, although there are a few reports on children and adolescents with T2D [4]. Most studies on adults with diabetes have produced conflicting data. Several studies have reported increased BMD in patients with T2D while other studies have shown decreased or unchanged bone mass in patients with T2D compared to normal controls [5-7]. Adolescence is a critical period of peak bone mass. T2D may affect bone density when it develops in adolescents. In addition, most patients with T2D suffer from impaired glucose tolerance, which can lead to increased resistance to insulin [8]. Thus, insulin resistance and T2D might lead to skeletal complications. 
The aim of this study was to assess BMD at multiple sites after correcting for several potential confounders such as age, sex, Tanner stage, and body mass index (BMI) known to affect BMD in children and adolescents with newly developed T2D.

\section{Subjects and Methods}

\section{Subjects}

This was a cross-sectional case-control study. A total of 17 obese children and adolescents with T2D were enrolled in this study. All patients were diagnosed with T2D at a single tertiary hospital between 2008 and 2014. The control group consisted of 59 healthy obese children and adolescents matched for age, sex, and body mass index (BMI) [9]. These control subjects were recruited from Seoul and Gyeonggi-do area where $50 \%$ of the Korean population resides. All participants were of the same ethnicity (Korean). Their parents had normal heights and weights. "Obese" was defined as having a BMI above the $85^{\text {th }}$ percentile for age and sex based on Korean national population standards. T2D was diagnosed according to the criteria of the ADA: 1) classic symptoms such as polyuria, polydipsia, and polyphagia, and random glucose level $\geq 200 \mathrm{mg} / \mathrm{dl}$, 2) fasting glucose level $\geq 126 \mathrm{mg} / \mathrm{dl}$, or 3) 2 -h plasma glucose level $\geq 200 \mathrm{mg} /$ $\mathrm{dl}$ after an oral glucose tolerance test (OGTT) [10]. All patients were negative for insulin autoantibody (IAA), glutamic acid decarboxylase antibodies (GADA), or islet cell antibody using radioimmunoassay (RSR, Cardiff, Wales, UK) at the time of initial diagnosis. Patients who had previously used medications such as glucocorticoids that might affect glucose and bone mineral metabolism were excluded. Subjects with genetic syndromes such as Prader-Willi syndrome and endocrine disorders were also excluded. No subjects had microvascular complications such as retinopathy, nephropathy, or neuropathy. No subjects had history of fracture.

Height, weight, bone age, and levels of insulin, serum glucose, calcium, phosphorus, alkaline phosphatase, and $\mathrm{HbA} 1 \mathrm{c}$ of patients were collected from their clinical charts at the time of evaluation. Height was measured using a Harpenden stadiometer (Holtain, Crosswell, Crymych, UK) and weight was recorded with a digital scale. BMI was calculated as weight in kilograms divided by height in meters squared. Standard deviation scores (SDS) of height, weight, and BMI were calculated according to the 2007 Korean National Growth Charts [11]. Pubertal status was assessed according to the Tanner and Marshall method for genital developmental status in boys and for breast development in girls [12].

\section{Laboratory measurements}

Of 17 patients, 11 children and adolescents underwent OGTT before treatment. After fasting for 12-h overnight, standard OGTT was done by ingestion of $1.75 \mathrm{~g} / \mathrm{kg}$ glucose to a maximum of $75 \mathrm{~g}$. One antecubital intravenous catheter was inserted for blood sampling. Its patency was maintained by slow infusion of normal saline. Blood samples were obtained every 30 min for 120 min to measure plasma glucose and insulin levels. Plasma glucose level was determined using a glucose analyzer by the glucose oxidase method (TBA200-FR, Toshiba, Japan). Plasma insulin was measured by radio immunoassay (Cobra II Gamma counter, Packard, USA). HbA1c was measured with turbidimetric inhibition immunoassay (COBAS Integra 800, Roche, Swiss). To assess insulin resistance, we used homeostasis model assessment of insulin resistance (HOMA-IR). HOMA-IR was calculated using the following formula: fasting insulin $(\mu \mathrm{U} / \mathrm{ml}) \times$ fasting glucose $(\mathrm{mmol} / \mathrm{l}) / 22.5$.

\section{Dual-energy X-ray absorptiometry}

Bone mineral density (BMD unit: grams $/ \mathrm{cm}^{2}$ ), bone mineral content (BMC unit: grams), and whole-body composition including lean body mass and fat mass were evaluated for all subjects using a Lunar Prodigy (General Electric, GE Healthcare, Madison, WI, USA). All patients underwent dual-energy X-ray absorptiometry (DEXA) within one week after the initial diagnosis. BMDs $\left(\mathrm{g} / \mathrm{cm}^{2}\right)$ at the level of lumbar spine segments L1-L4 (LS), femur neck (FN), and total body (TB) were measured with a whole-body scan. To adjust for body size, bone mineral apparent density (BMAD) was calculated using the following formula: $B M A D=B M D(L S) \times 4 /$ ( $\pi \times$ width) [13]. Z-scores for BMD at each site were calculated using data on healthy Korean children and adolescents ( 262 girls and 252 boys) after adjusting for height-for-age $[9,14]$. Subjects were carefully repositioned before every scan to minimize errors associated with changes in measurement geometry. Scans were taken by a single experienced operator. Variation coefficients for repeated measurement were $<1 \%$ of LS, FN, and TB. Ethical approval for this study was obtained from Ajou University Hospital Ethics Committee (AJIRB-MED-MDB-17-117). Written informed consent was obtained from all subjects and their parents.

\section{Statistical analysis}

Statistical analysis was performed using SPSS version 21.0 (IBM Corp., Armonk, NY, USA). Parameters (BMD Z-scores for LS, FN, TB, and whole-body composition) with normal distribution were evaluated with Kolmogorov-Smirnov test. One-way analysis of covariance (ANCOVA) was performed to assess differences between patients and controls after adjusting for age, sex, BMI, and Tanner stage. Pearson's correlation analysis was performed to determine the relationship between BMD Z-scores at each site and other clinical variables. When we found a significant association with BMD Z-scores at each site, linear regression was performed for multivariate analysis with stepwise variable selection, including age, BMI SDS, HbA1c, and HOMA-IR. Statistical significance was defined at $p<0.05$. All results are given as mean $\pm S D$, unless otherwise stated.

\section{Results}

The clinical characteristics of all subjects are shown in $>$ Table 1. The mean age of all subjects was $12.9 \pm 2.4$ years (range, $8.3-18.3$ years). Age, sex distribution, height SDS, weight SDS, or BMI SDS was not significantly different between the 2 groups. Mean $\mathrm{HbA} 1 \mathrm{C}$ level and HOMA-IR of patients were $95 \pm 3 \mathrm{mmol} / \mathrm{mol}$ and $9.7 \pm 6.4$, respectively. Serum C-peptide and insulin levels were $4.42 \pm 2.25 \mathrm{ng} /$ $\mathrm{ml}$ and $19.4 \pm 10.4 \mathrm{IU} / \mathrm{I}$, respectively. Of 17 patients with T2D, 8 patients were treated with hypoglycemic drugs at the time of initial diagnosis and 3 patients needed to take basal insulin and hypoglycemic drugs. Six patients were only treated with diet and exercise interventions. 
- Table 1 Comparison of clinical characteristics between patients with type 2 diabetes mellitus and obese controls in children and adolescents.

\begin{tabular}{|c|l|l|l|}
\hline Variable & $\begin{array}{l}\text { T2D } \\
(\mathbf{n = 1 7 )}\end{array}$ & $\begin{array}{l}\text { Obese controls } \\
(\mathbf{n = 5 9 )}\end{array}$ & $\mathbf{p}$-Value \\
\hline Age (years) & $13.5 \pm 2.0$ & $12.8 \pm 2.5$ & 0.202 \\
\hline Sex (M/F) & $10 / 7$ & $33 / 26$ & 0.529 \\
\hline Pubertal status & & & 0.353 \\
\hline Tanner stage 1 & 1 & 8 & \\
\hline Tanner stage 2 & 3 & 10 & \\
\hline Tanner stage 3 & 1 & 10 & \\
\hline Tanner stage 4 & 4 & 15 & \\
\hline Tanner stage 5 & 8 & 15 & 0.854 \\
\hline Height SDS & $0.46 \pm 1.02$ & $0.52 \pm 1.08$ & 0.409 \\
\hline Weight SDS & $1.83 \pm 1.67$ & $1.67 \pm 0.68$ & 0.462 \\
\hline BMI SDS & $1.89 \pm 0.57$ & $1.77 \pm 0.46$ & \\
\hline T2D: Type 2 diabetes mellitus. & & \\
\hline
\end{tabular}

\section{Bone mineral density}

After adjusting for age, sex, BMI SDS, and Tanner stage, BMD $\mathrm{ht}_{\mathrm{ht}}$ Z-scores for LS or TB were not significantly different between patients and controls. However, BMD $\mathrm{ht}_{\mathrm{ht}} \mathrm{Z}$-scores for $\mathrm{FN}$ and BMAD Z-scores for lumbar spines were significantly lower in type 2DM patients than those in healthy obese controls ( $\bullet$ Table 2 ). All subjects except one had bone density above -2.0 Z-scores. Low bone density of $L S$ (Z-score: $<-2$ ) was only found in one patient with T2D (Z-score: -3.17).

\section{Correlation analysis}

Univariate and multivariate analyses were performed to investigate the correlation between several variables (age, HbA1c, HOMA-IR, and BMI SDS) and BMD ${ }_{h t} Z$-scores at multiple sites. There were no significant correlations between these variables and BMD BMD ${ }_{\mathrm{ht}}$ Z-scores.

\section{Discussion}

In this study, $B M D_{h t} Z$-scores of $L S$ and $T B$ in adolescents with newly developed T2D were similar to those in healthy obese controls after adjusting for potential confounders. However, $B \mathrm{BD}_{\mathrm{ht}} \mathrm{Z}$-scores for FN and BMAD in patients with T2D were significantly lower than those in controls. HbA1c or insulin resistance was not associated with BMD Z-score.

It is well known that glucose and insulin levels can cause alterations in bone mineral metabolism in patients with types 1 and 2 DM [15]. There have been many studies on BMD in T2D in adults with inconsistent results. A meta-analysis has reported that patients with T2D have about 25-50\% SD higher BMD compared to normal control subjects [16]. In a recent review article on adults, most studies have reported increased BMD in patients with T2D
- Table 2 Bone mineral density (BMD) Z-scores for lumbar spine, femur neck, and total body in patients with type 2 diabetes and obese controls.

\begin{tabular}{|c|c|c|c|}
\hline Variable & $\begin{array}{l}\text { T2D } \\
(n=17)\end{array}$ & $\begin{array}{l}\text { Obese controls } \\
(n=59)\end{array}$ & p-Value ${ }^{\dagger}$ \\
\hline \multicolumn{4}{|l|}{ BMD $_{\text {ht }}$ Z-scores ${ }^{*}$} \\
\hline Lumbar spine & $-0.14 \pm 1.51$ & $0.27 \pm 0.80$ & 0.123 \\
\hline Femur Neck & $0.09 \pm 1.09$ & $0.65 \pm 0.95$ & 0.011 \\
\hline Total body & $0.36 \pm 0.89$ & $0.72 \pm 0.84$ & 0.069 \\
\hline BMAD Z-scores & $-0.41 \pm 1.16$ & $0.32 \pm 0.92$ & 0.012 \\
\hline $\begin{array}{l}\text { Lean body mass } \\
\text { Z-scores }\end{array}$ & $0.45 \pm 1.12$ & $1.21 \pm 1.20$ & 0.001 \\
\hline Fat mass Z-scores & $4.69 \pm 2.59$ & $3.33 \pm 2.73$ & 0.125 \\
\hline \multicolumn{4}{|c|}{$\begin{array}{l}\text { * The Z-scores for BMD at each site were calculated using data on } \\
\text { healthy Korean children and adolescents after adjusting for } \\
\text { height-for-age.; } † \text { One-way analysis of covariance (ANCOVA) was } \\
\text { performed to assess differences between patients and controls after } \\
\text { adjusting for age, sex, BMI and Tanner stage.; T2D: Type } 2 \text { diabetes } \\
\text { mellitus; BMAD: Bone mineral apparent density. }\end{array}$} \\
\hline
\end{tabular}

compared to healthy control subjects [17]. Thirteen studies revealed decreased BMD while 8 studies found no difference in BMD between patients and healthy controls [17]. These contradicting findings might be due to large variations in ethnicity, methods used for measuring BMD, sample size, and duration of disease. In our study, BMDs of $L S$ and TB except $F N$ in patients with T2D were not significantly different from those in the control group. It is currently unclear why BMDs of FN only are lower in patients with T2D than those in controls. A previous study has reported that the higher the ratio of cortical and trabecular bone (distal radius $>$ femoral neck > lumbar spine), the lower the BMD Z-score (lumbar spine $>$ femoral neck $>$ distal radius) in women with T2D [6]. Nakamura et al. [18] have also reported that T2D patients have significantly lower cortical bone thickness, but not trabecular BMD, compared to non-diabetic controls. These results suggest that selective loss of cortical bone might have influenced different bone density at multiple bony sites.

Higher BMI has been identified as a strong determinant of higher BMD [19]. Thus, a comparison between patients and BMImatched controls may be important. In our study, patients with T2D in the early period after diagnosis showed no differences in BMD in comparison with BMI-matched controls, although many studies reported higher BMD in DM patients after adjusting for BMI [16]. There have been no reports on BMD in pediatric patients with T2D. Afghani et al. [20] have compared BMDs of children with impaired glucose tolerance to those in children with normal glucose tolerance. They reported that BMD was not significantly different between pre-diabetic overweight children and normal overweight controls. 
The pathogenesis of bone disease in patients with T2D includes multiple factors. The early phase is characterized by hyperinsulinemia or insulin resistance whereas the late phase is characterized by the development of vascular complications [21]. In an experimental study, insulin can stimulate osteoblast proliferation and insulin-mediated signals in osteoblasts can activate bone resorption markers [22]. In addition to direct effects of insulin on bone, hyperinsulinemia could mediate bone metabolism by changing the IGF-1 axis [23]. Although results regarding the effect of insulin resistance or hyperinsulinemia on BMD have been inconsistent, Abrahamsen et al. have reported that low insulin sensitivity is correlated with high BMD in healthy men [24]. A recent study by Arikan et al. [25] has reported that insulin resistance has a negative effect on BMD in patients with type $2 \mathrm{DM}$. In our study, insulin resistance was not significantly associated with BMD in patients with T2D.

Patients with T2D have an increased risk of fracture regardless of increased or normal BMD [26]. In the Rotterdam study [27], adults above 55 years of age with T2D have increased risk of nonvertebral fracture (hazard ratio: 1.33). Although several mechanisms have been proposed for diabetes-related bone fracture, the exact mechanism remains largely unclear. Petit et al. [28] have reported that older men with T2D have smaller total bone area at the tibia than controls based on peripheral quantitative computed tomography. In the MINOS study [29], small bone width is associated with incident fractures in elderly men. In a recent study, disc height has been found to be lower in patients with T2D than that in controls [30]. Decreased bone size may be associated with fracture because decreased bone strength can result in inability to withstand mechanical loads. In our study, BMAD of the lumbar spine in patients with T2D was significantly lower than that in controls. Thus, the size of bone should be taken into account when analyzing BMD in patients with $\mathrm{T} 2 \mathrm{D}$.

Our study has several limitations stemming from its case-control study design. First, we did not evaluate markers related to bone metabolism, calcium intake, or physical activity. Thus, it was difficult to determine causality. Second, the sample size was very small because the prevalence of T2D in younger individuals was low in Korea. In spite of these limitations, to the best of our knowledge, this is one of the first studies to measure BMD and body composition in children and adolescents with newly developed T2D.

In conclusion, our study revealed that $\mathrm{BMD}_{\mathrm{ht}} \mathrm{Z}$-scores at multiple sites except FN in children and adolescents with T2D were not different from those in obese controls. Longitudinal and large cohort studies are needed in the future to validate the association between diabetes and bone metabolism using more accurate methods to assess bone width such as peripheral quantitative computerized tomography.

\section{Conflict of Interest}

The authors declare that they have no conflict of interest. We confirm that we have read the Journal's position on issues involving ethical publication and affirm that this report is consistent with those guidelines.
References

[1] Wang Y, Lobstein T. Worldwide trends in childhood overweight and obesity. Int J Pediatr Obes 2006; 1: 11-25

[2] Kim HM, Park J, Kim HS, Kim DH, Park SH. Obesity and cardiovascular risk factors in korean children and adolescents aged $10-18$ years from the korean national health and nutrition examination survey, 1998 and 2001. Am J Epidemiol 2006; 164: 787-793

[3] Leslie WD, Rubin MR, Schwartz AV, Kanis JA. Type 2 diabetes and bone. J Bone Mineral Res 2012; 27: 2231-2237

[4] Chobot AP, Haffke A, Polanska J, Halaba ZP, Deja G, Jarosz-Chobot P, Pluskiewicz W. Bone status in adolescents with type 1 diabetes. Diabetologia 2010; 53: 1754-1760

[5] Yaturu S, Humphrey S, Landry C, Jain SK. Decreased bone mineral density in men with metabolic syndrome alone and with type 2 diabetes. Med Sci Monitor 2009; 15: Cr5-Cr9

[6] Majima T, Komatsu Y, Yamada T, Koike Y, Shigemoto M, Takagi C, Hatanaka I, Nakao K. Decreased bone mineral density at the distal radius, but not at the lumbar spine or the femoral neck, in Japanese type 2 diabetic patients. Osteoporos Int 2005; 16: 907-913

[7] Dennison EM, Syddall HE, Aihie Sayer A, Craighead S, Phillips DI, Cooper C. Type 2 diabetes mellitus is associated with increased axial bone density in men and women from the Hertfordshire cohort Study: Evidence for an indirect effect of insulin resistance? Diabetologia 2004; 47: 1963-1968

[8] Unwin N, Shaw J, Zimmet P, Alberti KG. Impaired glucose tolerance and impaired fasting glycaemia: The current status on definition and intervention. Diabetic Med 2002; 19: 708-723

[9] Lim JS, Hwang JS, Lee JA, Kim DH, Park KD, Cheon G], Shin CH, Yang SW. Bone mineral density according to age, bone age, and pubertal stages in korean children and adolescents. J Clin Densitom 2010; 13: 68-76

[10] [Anonymous]. Standards of medical care in diabetes-2014. Diabetes Care 2014; 37 (Suppl 1): S14-S80

[11] Moon JS, Lee SY, Nam CM, Choi JM, Choe BK, Seo JW, Oh K, Jang MJ, Hwang SS, Yoo MH, Kim YT, Lee CG. 2007 Korean national growth charts: Review of developmental process and an outlook. Korean J Pediatr 2008; 51: 1-25

[12] Wheeler MD. Physical changes of puberty. Endocrinol Metab Clin North Am 1991; 20: 1-14

[13] Kroger H, Vainio P, Nieminen J, Kotaniemi A. Comparison of different models for interpreting bone mineral density measurements using DXA and MRI technology. Bone 1995; 17: 157-159

[14] Zemel BS, Leonard MB, Kelly A, Lappe JM, Gilsanz V, Oberfield S, Mahboubi S, Shepherd JA, Hangartner TN, Frederick MM, Winer KK, Kalkwarf HJ. Height adjustment in assessing dual energy $x$-ray absorptiometry measurements of bone mass and density in children. J Clin Endocrinol Metab 2010; 95: 1265-1273

[15] Napoli N, Chandran M, Pierroz DD, Abrahamsen B, Schwartz AV, Ferrari SL. Mechanisms of diabetes mellitus-induced bone fragility. Nat Rev Endocrinol 2017; 13: 208-219

[16] Ma L, Oei L, Jiang L, Estrada K, Chen H, Wang Z, Yu Q, Zillikens MC, Gao X, Rivadeneira F. Association between bone mineral density and type 2 diabetes mellitus: a meta-analysis of observational studies. Eur J Epidemiol 2012; 27: 319-332

[17] Abdulameer SA, Sulaiman SA, Hassali MA, Subramaniam K, Sahib MN. Osteoporosis and type 2 diabetes mellitus: What do we know, and what we can do? Patient Pref Adher 2012; 6: 435-448

[18] Nakamura M, Inaba M, Yamada S, Ozaki E, Maruo S, Okuno S, Imanishi Y, Kuriyama N, Watanabe Y, Emoto M, Motoyama K. Association of decreased handgrip strength with reduced cortical thickness in japanese female patients with type 2 diabetes mellitus. Sci Rep 2018; 8: 10767 
[19] Vestergaard P. Discrepancies in bone mineral density and fracture risk in patients with type 1 and type 2 diabetes-a meta-analysis. Osteoporos Int 2007; 18: 427-444

[20] Afghani A, Cruz ML, Goran MI. Impaired glucose tolerance and bone mineral content in overweight latino children with a family history of type 2 diabetes. Diabetes Care 2005; 28: 372-378

[21] Shanbhogue VV, Mitchell DM, Rosen C], Bouxsein ML. Type 2 diabetes and the skeleton: New insights into sweet bones. Lancet Diabetes Endocrinol 2016; 4: 159-173

[22] Ferron M, Wei ], Yoshizawa T, Del Fattore A, DePinho RA, Teti A, Ducy P, Karsenty $G$. Insulin signaling in osteoblasts integrates bone remodeling and energy metabolism. Cell 2010; 142: 296-308

[23] Jehle PM, Jehle DR, Mohan S, Bohm BO. Serum levels of insulin-like growth factor system components and relationship to bone metabolism in Type 1 and Type 2 diabetes mellitus patients. J Endocrinol 1998; 159: 297-306

[24] Abrahamsen B, Rohold A, Henriksen JE, Beck-Nielsen H. Correlations between insulin sensitivity and bone mineral density in non-diabetic men. Diabetic Med 2000; 17: 124-129
[25] Arikan S, Tuzcu A, Bahceci M, Ozmen S, Gokalp D. Insulin resistance in type 2 diabetes mellitus may be related to bone mineral density. J Clin Densitom 2012; 15: 186-190

[26] Starup-Linde J, Frost M, Vestergaard P, Abrahamsen B. Epidemiology of fractures in diabetes. Calcif Tissue Int 2017; 100: 109-121

[27] de L II, van der Klift M, de Laet CE, van Daele PL, Hofman A, Pols HA. Bone mineral density and fracture risk in type- 2 diabetes mellitus: The rotterdam study. Osteoporos Int 2005; 16: 1713-1720

[28] Petit MA, Paudel ML, Taylor BC, Hughes JM, Strotmeyer ES, Schwartz AV, Cauley JA, Zmuda JM, Hoffman AR, Ensrud KE. Bone mass and strength in older men with type 2 diabetes: The Osteoporotic Fractures in Men Study. J Bone Mineral Res 2010; 25: 285-291

[29] Szulc P, Munoz F, Duboeuf F, Marchand F, Delmas PD. Low width of tubular bones is associated with increased risk of fragility fracture in elderly men-the MINOS study. Bone 2006; 38: 595-602

[30] Agius R, Galea R, Fava S. Bone mineral density and intervertebral disc height in type 2 diabetes. J Diabetes Complicat 2016; 30: 644-650 